This item was submitted to Loughborough's Research Repository by the author.

Items in Figshare are protected by copyright, with all rights reserved, unless otherwise indicated.

Computation of ground vibrations generated by accelerating and braking road vehicles

PLEASE CITE THE PUBLISHED VERSION

http://dx.doi.org/10.1177/107754639600200303

PUBLISHER

(c) Sage

VERSION

AM (Accepted Manuscript)

LICENCE

CC BY-NC-ND 4.0

REPOSITORY RECORD

Krylov, Victor V.. 2013. "Computation of Ground Vibrations Generated by Accelerating and Braking Road Vehicles”. figshare. https://hdl.handle.net/2134/11361. 
This item was submitted to Loughborough's Institutional Repository (https://dspace.lboro.ac.uk/) by the author and is made available under the following Creative Commons Licence conditions.

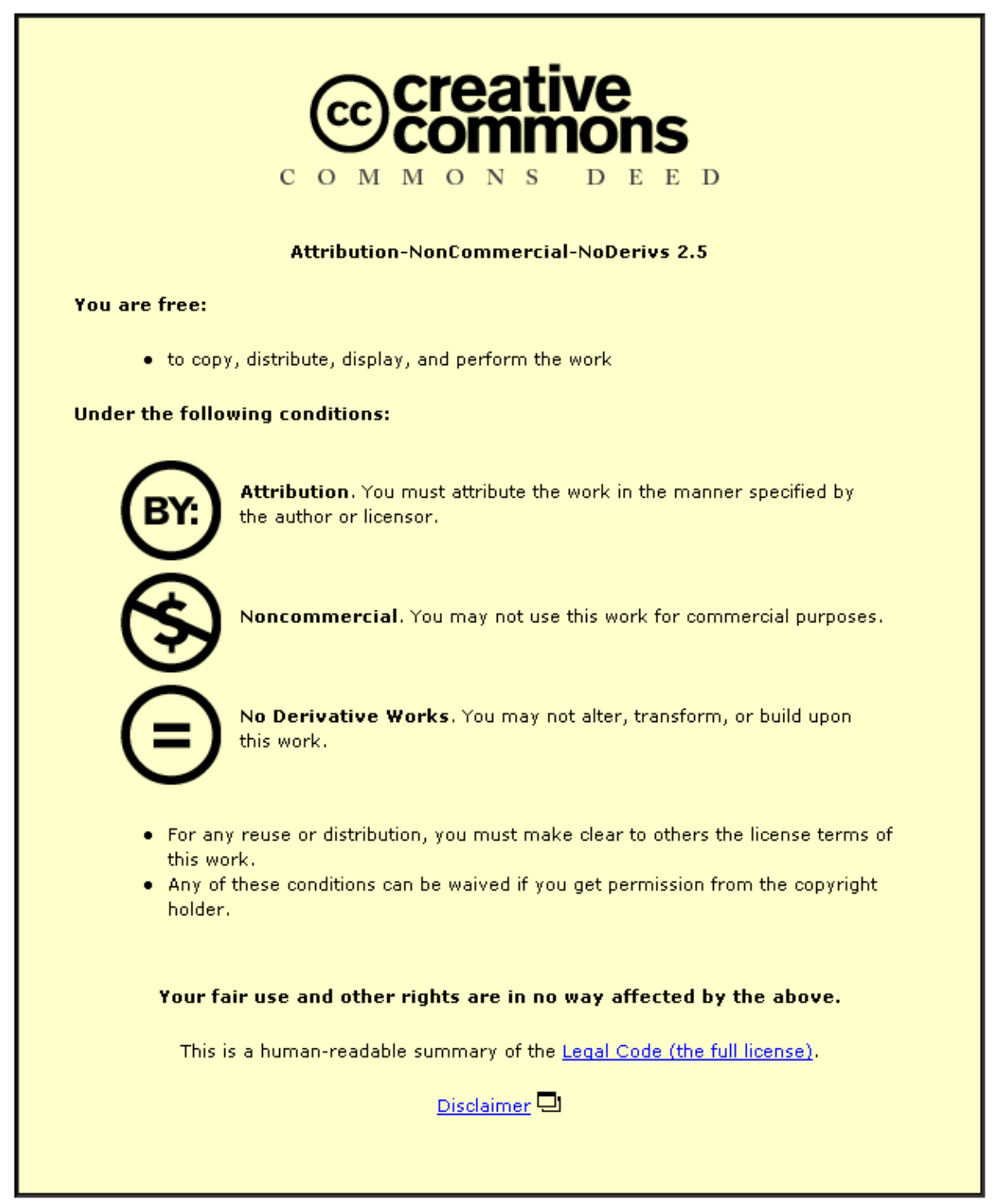

For the full text of this licence, please go to: http://creativecommons.org/licenses/by-nc-nd/2.5/ 


\title{
COMPUTATION OF GROUND VIBRATIONS GENERATED BY ACCELERATING AND BRAKING ROAD VEHICLES
}

\author{
VICTOR V. KRYLOV \\ Centre for Research into the Built Environment, Nottingham Trent University, \\ Burton Street, Nottingham NG1 4BU, UK
}

Abstract: The problem of ground vibration generation by accelerating and braking road vehicles is considered theoretically for vehicles accelerating (decelerating) with a constant acceleration $a$ from rest to a constant speed, or braking to a stop from a constant speed. According to the low-frequency approximation considered, an accelerating or braking vehicle of mass $M$ is modelled as a point horizontal traction force $F_{X}=a M$ applied to the ground and moving along with the vehicle. Frequency spectra of the vertical component of the ground vibration velocity are investigated as functions of acceleration, final (initial) speed of the vehicle, ground attenuation, Poisson ratio, and radiation angle $\Theta$ relative to the direction of the vehicle movement. It is shown that, in contrast to vehicles travelling on uneven roads, the ground vibrations generated by accelerating and braking vehicles are characterised by directivity patterns $V(\Theta)$ which show, in particular, that there is no radiation in the directions perpendicular to the direction of vehicle movement.

Key words: Ground vibrations, generation, road vehicles 


\section{List of Notations}

$a \quad$ vehicle acceleration (deceleration)

$c_{l, t} \quad$ phase velocities of bulk longitudinal and shear waves

$c_{R} \quad$ Rayleigh wave velocity

$F \quad$ frequency

$F(k)$ Rayleigh determinant

$F_{X} \quad$ horizontal traction force

$G_{x i}$ components of the dynamic Green's tensor

$\boldsymbol{k} \quad$ surface projection of a wave vector

$k_{l, t} \quad$ wavenumbers of bulk longitudinal and shear waves

$k_{R} \quad$ wavenumber of Rayleigh waves

$M \quad$ vehicle mass

$t \quad$ time

$T_{x z} \quad$ component of a load stress tensor

$\boldsymbol{u}$ displacement vector

$u_{i} \quad$ components of the displacement vector

$u_{i j} \quad$ components of the strain tensor

$v \quad$ final (initial) vehicle speed

$V \quad$ vertical ground vibration amplitude

$v_{z} \quad$ vertical component of the ground vibration velocity

$x, y, z$ space coordinates

$\gamma \quad$ attenuation constant of the ground

$\omega \quad$ circular frequency

$\Theta \quad$ observation angle

$\sigma \quad$ Poisson ratio

$\rho$ surface radius-vector

$\lambda, \mu$ elastic Lamé constants

$\rho_{0} \quad$ mass density of the ground

$\sigma_{i j} \quad$ components of the elastic stress tensor

$v_{l, t} \quad$ constants of Rayleigh wave attenuation into the ground 


\section{Introduction}

Generation of ground vibrations by road vehicles, in particular by heavy lorries, may result in significant nuisance to residents living in nearby properties, fear of possible property damage, and damage to road surfaces. Understanding basic mechanisms of generating ground vibrations by cars and lorries can provide useful information for road design and residential planning that would assist in reducing ground vibration intensity at sensitive locations.

According to the survey of vibration nuisance from road traffic undertaken by the UK Transport Research Laboratory (Watts 1984), among the most annoying mechanisms of traffic-induced ground vibrations are: 1) vehicles accelerating and braking - respectively $49.7 \%$ and $30.2 \%$ of the respondents, and 2 ) damaged or bumpy road surfaces $-39.4 \%$ of the respondents.

Ground vibrations due to damaged and uneven road surfaces have been investigated both analytically and numerically by several authors (e.g., Taniguchi and Sawada 1979, le Houedec and Riou 1982, Hunt 1991, Hanazato et al. 1991). In the paper by Hunt (1991) a statistical description of normal load forces was developed taking into account excitation of the main vehicle resonances and their influence on generation of ground vibrations. In the paper by Hanazato et al. (1991), which concentrated on numerical 
calculation of ground vibrations generation on real complex road structure, a simpler dynamical force modelling was used that did not take account of vehicle resonances.

Despite the reported significance of ground vibration radiation caused by accelerating or braking (Watts 1984), there is no adequate theoretical analysis of vibrations from accelerating or braking vehicles.

The existing literature on elastic wave radiation by forces moving at nonconstant speed along a free surface is not directly applicable to the case of road-traffic-induced ground vibrations. For example, Beitin (1969) considered the response of an elastic half space to a decelerating surface point load, directed normally to the surface and associated with blast waves generated by a nuclear explosion. The load speed varied from superseismic to a value slightly less than the Rayleigh surface wave velocity. In contrast, road vehicle speeds are invariably much lower than Rayleigh wave velocities. Note in this connection, that trans-Rayleigh train speeds are a reality and have important implications for generation of ground vibrations (Krylov 1995).

It should also be emphasised that, in contrast to the normal load forces considered by Beitin (1969), the main mechanism of ground vibration generation by accelerating or decelerating vehicles is the action of horizontal traction forces which are applied from tyres to the ground only during the time of accelerating or braking. The contribution of normal load forces is relatively small for speeds and accelerations typical for road traffic since for ideally flat surfaces these forces are time-independent. 
In the present paper we consider generation of ground vibrations by vehicles accelerating at constant acceleration $a$ from rest to a constant speed $v$, or braking with constant acceleration $-a$ from $v$ to a complete stop. The assumption of constant acceleration (deceleration) is in agreement with general requirements for vehicle performance, e.g., in the process of braking. According to these requirements (Newcomb and Spurr 1967), deceleration produced during braking should ideally be uniform throughout an application. Note that during deceleration horizontal interaction forces, acting during deceleration between vehicle and road through the tyre-road interface, are limited by friction (adhesion) between tyre and road. If the product of deceleration and vehicle mass exceeds this friction, sliding or skidding will occur. Road braking statistics show (Newcomb and Spurr 1967) that in the majority of cases decelerations range from 0.1 to $0.2 \mathrm{~g}$, i.e. from about 1 to $2 \mathrm{~m} / \mathrm{s}^{2}$. However, in emergency braking decelerations of 0.4- $0.6 \mathrm{~g}$ are typical, and greater than $1.0 \mathrm{~g}$ is possible.

Throughout this paper, we consider vehicle acceleration $\pm a$ as a given value resulting from joint application of engine or braking forces, drag forces caused by wind resistance, rolling resistance, friction in the wheel bearings etc (Newcomb and Spurr 1967, Wong 1993). We also assume that the most important internal degrees of freedom of the vehicle (corresponding to the pitch, bounce and wheel resonances (Wong 1993)) are not excited by horizontal inertial forces produced during acceleration or deceleration.

Being interested only in the low-frequency range of generated ground vibrations, we will model an accelerating or breaking vehicle of mass $M$ as a point horizontal traction 
force $F_{X}=a M$ applied to the ground and moving along the road at vehicle speed. For braking or accelerating respectively the direction of this force coincides with the direction of vehicle movement or is opposite to it. We also assume that adhesion between road and tyres is perfect. Thus, the limitations on acceleration (deceleration) caused by sliding or skiding will not be considered. Note that for the ground vibration generation mechanism associated with uneven surfaces the equivalent dynamic load forces applied to the ground are directed perpendicular to the ground surface (e.g., Hunt 1991, Hanazato et al. 1991).

The solution of the problem under consideration is sought using the Green's function method similar to that described by Krylov and Ferguson (1994). The resulting ground vibration field is then calculated for the far zone of radiation taking into account only the Rayleigh surface wave contribution.

Two approaches to the calculation of the Fourier integral for the load forces are discussed: an approximate approach that ignores changing location of the vehicle during acceleration (deceleration), and a more exact one that takes location into account. The approximate approach allows the problem to be simplified significantly since the resulting Fourier integral is easily expressed analytically. In contrast, the numerical calculation of the exact integral demands much more computation. It is shown, however, that although ground vibration velocities $V$ calculated using these approaches are essentially different if rigorous (narrow band) Fourier spectra are being calculated, they become almost identical in terms of $1 / 3$-octave averaged spectra traditionally used in 
practical measurements in environmental acoustics. This allows one to use for practical calculations much simpler approximate expressions.

Using this simplification, the 1/3-octave frequency spectra and 1/3-octave averaged amplitudes of ground vibrations generated by heavy lorries $(M=20000 \mathrm{~kg})$ are investigated as functions of acceleration $\pm a$, the final or initial speed of the vehicle $v$, ground attenuation constant $\gamma$, Poisson ratio $\sigma$, and radiation angle $\Theta$ relative to the direction of vehicle movement. In the final part of the paper, some conclusions following from the results are discussed.

\section{Governing Equations}

Being interested only in the low-frequency ground vibrations typical of traffic-induced mechanisms of generation, we will model a vehicle as a point load moving along the surface either with acceleration or deceleration. The low-frequency approximation assumes that the shortest wave-lengths of the generated ground vibration spectra are larger than the dimensions of the vehicle. This is usually satisfied for all frequencies of traffic-induced ground vibration spectra, which have an upper limit around $30 \mathrm{~Hz}$ (e.g., Hunt 1991). In fact, the resulting point force which is being used in the model is the sum of partial forces applied to the ground from each wheel of the vehicle. 
If a vehicle is accelerated or decelerated at constant absolute value $a$, then the point load force applied from a vehicle to the ground surface is a horizontal traction force that moves along the x-axis with a vehicle (see Figure 1) and has amplitude

$$
F_{X}=a M
$$

The related mechanical shear stresses applied to the ground surface during acceleration or deceleration, i.e. during the period of time from $t=0$ to $t=v / a$, where $v$ is the final or initial speed, are described respectively as follows:

$$
T_{x z}(\rho, t)=-F_{x} \delta\left(x-a t^{2} / 2\right) \delta(y),
$$

and

$$
T_{x Z}(\rho, t)=F_{X} \delta\left(x-v t+a t^{2} / 2\right) \delta(y) .
$$

Here $T_{i j}$, where $i, j=x, y, z$, are the components of a load stress tensor applied to the surface, $\rho=\{x, y\}$ is the surface radius-vector, and $\delta(y)$ is Dirac's delta-function.

The ground vibration field generated by accelerating or braking vehicles in an elastic half space, which we will assume to be homogeneous and isotropic, should satisfy the elastic Lame' equation 


$$
(\lambda+2 \mu) \operatorname{grad} \operatorname{div} \boldsymbol{u}-\mu \operatorname{rot} \operatorname{rot} \boldsymbol{u}-\rho_{0} \partial^{2} \mathbf{u} / \partial^{2}=0,
$$

and the boundary conditions on the ground surface taking into account only horizontal traction forces (2),(3):

$$
\begin{gathered}
\sigma_{x z}=2 \mu u_{x z}=-T_{x z}(\rho, t), \\
\sigma_{y z}=2 \mu u_{y z}=0, \\
\sigma_{z z}=\lambda u_{n n}+2 \mu u_{z z}=0 .
\end{gathered}
$$

Here $\boldsymbol{u}$ is the particle displacement vector with the components $u_{i} ; \lambda$ and $\mu$ are the elastic Lame' constants; $\rho_{0}$ is the ground mass density; and $u_{i j}=(1 / 2)\left(\partial u_{i} / \partial \mathrm{x}_{\mathrm{j}}+\partial u_{j} / \partial \mathrm{x}_{i}\right)$ are the components of the linearised strain tensor.

We wish to solve the problem described by equations (2)-(5) in the far zone of radiation relative to the characteristic wavelengths of generated ground vibrations and the acceleration or braking distance. Without loss of generality, we will also limit our calculation of frequency spectra to the vertical component of ground vibration velocity $v_{z}$ $=d u_{z} / d t$ which is usually determined in experimental observations. 


\section{Formal Solution}

The formal solution to the problem (2)-(5) may be written as a Fourier integral

$$
u_{i}(\boldsymbol{r}, t)=\left(1 / 4 \pi^{2}\right) \int_{-\infty}^{\infty} \int_{-\infty-\infty}^{\infty} \int_{x z}^{\infty} T_{x z}(\omega, \boldsymbol{k}) G_{x i}(\omega, \boldsymbol{k}, z) e^{-i \omega t+i(\boldsymbol{k} \rho)} d \omega d \boldsymbol{k},
$$

where $\boldsymbol{r}=\{x, y, z\}$ is the three-dimensional radius-vector of the observation point, $\boldsymbol{k}=$ $\left\{k_{x}, k_{y}\right\}$ is the projection of a current three-dimensional wave-vector onto the plane $z=0$, and $\omega$ is the circular frequency. Functions $T_{x z}(\omega, \boldsymbol{k})$ and $G_{x i}(\omega, \boldsymbol{k}, z)$ are respectively the Fourier transforms of the corresponding components of a load force source and the dynamic Green’s tensor (Green’s function) which are determined by the following formulae:

$$
T_{x z}(\omega, \boldsymbol{k})=(1 / 2 \pi) \int_{0}^{\mathrm{v} / \mathrm{a}} \int_{-\infty-\infty}^{\infty} \int_{-\infty}^{\infty} T_{x z}(x, y, t) e^{i \omega t-i(\boldsymbol{k} \rho)} d \rho d t
$$

and

$$
G_{x i}(\omega, \boldsymbol{k}, z)=(1 / 2 \pi) \int_{-\infty-\infty-\infty}^{\infty} \int_{-\infty}^{\infty} \int_{x i}(\rho, t, z) e^{i \omega t-i(\boldsymbol{k} \rho)} d \rho d t .
$$


We remind the reader that the Green's function $G_{x i}(\rho, t, z)$ describes the outgoing waves resulting from the solution of the differential equation (4) in the elastic half space subject to the boundary conditions

$$
\begin{gathered}
\sigma_{x z}=2 \mu u_{x z}=-\delta(\rho-\rho) \delta(t-t), \\
\sigma_{y z}=2 \mu u_{y z}=0 \\
\sigma_{z z}=\lambda u_{n n}+2 \mu u_{z z}=0 .
\end{gathered}
$$

This function is invariant with translations in time and space (in directions parallel to the free surface), i.e., it is not changed if $t$ and $\rho$ are replaced by $t-t^{\prime}$ and $\rho-\rho^{\prime}$ respectively. Its physical sense is that it describes the total elastic field generated at the moment of time $t$ in the point $\boldsymbol{r}=\{\rho, z\}$ of the elastic half space by the impulsive horizontal point force applied at the moment $t^{\prime}<t$ (in agreement with the causality principle) to the point $\rho^{\prime}$ on the surface.

The solution of this and related problems for different load forces, whether using the terminology of Green's function or otherwise, has been considered by many authors beginning with Lamb (1904). Methods of solution of Lamb’s problem, both in time and frequency domains, are reviewed in a number of books (e.g., Ewing et al. 1957, Graff 1975). 
The Fourier transform of the relevant component $G_{x z}(\omega, \boldsymbol{k}, z)$ of the dynamic Green's tensor for an elastic half space is:

$$
G_{x z}(\omega, \boldsymbol{k}, z)=i \frac{2 k_{x} v_{l} v_{t}}{\mu F(k)} e^{-v_{l} z}+i \frac{k_{x}}{\mu F(k) k_{t}^{2}}\left[F(k)-2 k^{2} b(k)\right] e^{-v_{t} z} .
$$

Here the following notation is used: $k=\left(k_{x}^{2}+k_{y}^{2}\right)^{1 / 2}, b(k)=2 k^{2}-k_{t}^{2}-2 v_{l} v_{t}, \quad v_{l, t}=\left(k^{2}-\right.$ $\left.k_{l, t}^{2}\right)^{1 / 2} ; k_{l, t}=\omega / c_{l, t}$ are the wavenumbers of bulk longitudinal and shear acoustic waves; $c_{l}=\left[(\lambda+2 \mu) / \rho_{0}\right]^{1 / 2}$ and $c_{t}=\left(\mu / \rho_{0}\right)^{1 / 2}$ are their phase velocities; $F(k)=\left(2 k^{2}-k_{t}^{2}\right)^{2}-$ $4 k^{2} v_{l} v_{t}$ is the so called Rayleigh determinant.

Using equation (10) in (6) and applying the equality $v_{z}(\boldsymbol{r}, \omega)=-i \omega \boldsymbol{u}_{z}(\boldsymbol{r}, \omega)$, one can easily determine the spectrum of the vertical component of the ground vibration velocity:

$$
v_{z}(\boldsymbol{r}, \omega)=-i \omega\left(1 / 4 \pi^{2}\right) \int_{-\infty-\infty}^{\infty} \int_{x z}^{\infty} T_{x}(\omega, \boldsymbol{k}) G_{X Z}(\omega, \boldsymbol{k}, z) e^{-i \omega t+i(\boldsymbol{k} \rho)} d \boldsymbol{k} .
$$

Integration over $\boldsymbol{k}$ in (11) may be performed in polar co-ordinates $k, \varepsilon$ in corresponding planes of complex variables $k$ and $\varepsilon$. Then the residue at $k=k_{R}$, where $k_{R}$ is the wavenumber of a Rayleigh surface wave, along with the contribution of the saddle points during the integration over polar angle $\varepsilon$ give the elastic field of generated 
Rayleigh surface waves. Note that $k_{R}$ is determined as a real root of the equation $F(k)=$ 0 which also determines the velocity of Rayleigh waves: $c_{R}=\omega / k_{R}$.

The combined contributions of the saddle points in the complex planes $k$ and $\varepsilon$ give the far-field zone of generated longitudinal and shear bulk acoustic waves. Note, however, that the contribution of bulk waves to the ground vibration field generated on the surface $z=0$, which is of primary interest here, is proportional to $\left(k_{l} \rho\right)^{-2}$ and to $\left(k_{t} \rho\right)^{-2}$ respectively for longitudinal and shear waves. For comparison, the contribution of Rayleigh waves is proportional only to $\left(k_{R} \rho\right)^{-1 / 2}$. Therefore, in further calculations we will take into account only the contribution of Rayleigh surface waves into the vertical component of ground vibration velocity. We recall that the horizontal component associated with Rayleigh surface waves is of the same order of magnitude (e.g., Graff 1975).

Until now we did not consider attenuation of generated ground vibrations while propagating through the ground. The easiest way to take this into account is to replace the wavenumber of a Rayleigh wave in an ideal elastic medium $k_{R}=\omega / c_{R}$ by the complex wavenumber $k_{R}=k_{R}(1+i \gamma)=\left(\omega / c_{R}\right)(1+i \gamma)$, where $\gamma<<1$ is a positive constant which describes the linear dependence of a Rayleigh wave attenuation coefficient on frequency. According to Gutovdki and Dym (1976), for different types of ground the values of $\gamma$ are in the range from 0.01 to 0.2 . 
Taking all the above-mentioned into account, we can write the final expression for the vertical component of the ground vibration velocity $v_{z}(r, \omega)$ in the form

$$
\begin{aligned}
v_{Z}(\mathbf{r}, \omega)= & \left(\frac{2 \pi}{k_{R} \rho}\right)^{1 / 2} \frac{\omega k_{R}^{2}\left[k_{t}^{2} v_{l} v_{t}-k_{R}^{2} b\left(k_{R}\right)\right] \cos \Theta}{\pi \mu F^{\prime}\left(k_{R}\right) k_{t}^{2}} \times \\
& T_{X Z}\left(\omega, k_{R} \cos \Theta\right) e^{-k_{R} \gamma \rho} e^{i k_{R} \rho-i 3 \pi / 4}
\end{aligned}
$$

where $\Theta=\cos ^{-1}(x / \rho)$ is the observation angle and $F^{\prime}\left(k_{R}\right)$ is the derivative $d F(k) / d k$ taken at $k=k_{R}$.

Let us now discuss in more detail the Fourier transforms $T_{x z}\left(\omega, k_{R} \cos \theta\right)$ of the load forces (equations (2) and (3)). Substituting equations (2) and (3) into equation (7) and performing integration over $x$ and $y$, taking into account properties of the delta-function, one can obtain the following expressions for accelerating and braking vehicles respectively:

$$
\begin{aligned}
& T_{x z}\left(\omega, k_{R} \cos \theta\right)=-(1 / 2 \pi) a M \int_{0}^{\mathrm{v} / \mathrm{a}} \mathrm{e}^{\mathrm{i} \omega \mathrm{t}-\mathrm{ik}_{\mathrm{R}} \cos \Theta\left(\mathrm{at}^{2} / 2\right)} \mathrm{dt}, \\
& T_{x z}\left(\omega, k_{R} \cos \theta\right)=(1 / 2 \pi) a M \int_{0}^{\mathrm{v} / \mathrm{a}} \mathrm{e}^{\mathrm{i}\left(\omega-\mathrm{k}_{\mathrm{R}} \mathrm{v} \cos \Theta\right) \mathrm{t}+\mathrm{ik}_{\mathrm{R}} \cos \Theta\left(\mathrm{at}^{2} / 2\right)} \mathrm{dt} .
\end{aligned}
$$


Taking these integrals analytically gives very bulky expressions containing the so called error functions of the complex variable $\operatorname{erf}(\mathrm{z})$, which are very inconvenient for analysis and numerical computations. For brevity these expressions are not given here. The only useful conclusion derived from these expressions follows from their comparison. Such a comparison shows that they differ from each other only by the phase factor. Thus, if one is interested only in the amplitudes of generated vibrations, it is sufficient to consider just one of these cases, e.g. the case of braking vehicles.

\section{Approximate Approach}

The complexity of the integrals (13) and (14) justifies an approximate analytical evaluation avoiding direct numerical integration. If the approximate values are reasonably close to those derived by exact numerical calculation they can be recommended for practical use and will save much computation time.

One of the simplest approximate approaches is based on neglecting the exact space location of the vehicle during acceleration or braking; this reduces the more complex radiation problem for a moving source to the simplified one of a stationary source acting

during the period of time from $t=0$ to $t=v / a$. For example, in the case of a braking vehicle the corresponding approximation means that equation (14) is replaced by 


$$
T_{X z}\left(\omega, k_{R} \cos \theta\right)=T_{x z}(\omega)=(1 / 2 \pi) a M \int_{0}^{\mathrm{v} / \mathrm{a}} \mathrm{e}^{\mathrm{i} \omega \mathrm{t}} \mathrm{dt}=\frac{1}{2 \pi} v M \frac{\sin (\omega \nu / 2 a)}{\omega \nu / 2 a} e^{i \omega v / 2 a} .
$$

Let us compare the results of numerical calculations of the vertical ground vibration amplitude spectra $V(\rho, \omega)=\left|v_{z}(\rho, \omega)\right|$, where $v_{z}(\rho, \omega)$ is determined according to equation (12) using respectively equations (15) and (14) for $T_{x z}\left(\omega, k_{R} \cos \theta\right)$. Calculations were carried out for a braking lorry with a mass $M=20000 \mathrm{~kg}$ at the observation distance $\rho=50 \mathrm{~m}$ and angle $\Theta=\pi / 3$. Mass density of the ground $\rho_{0}$ was $2000 \mathrm{~kg} / \mathrm{m}^{3}$, velocity of longitudinal bulk waves $-c_{l}=471 \mathrm{~m} / \mathrm{s}$, shear waves $-c_{t}=272 \mathrm{~m} / \mathrm{s}$ and Rayleigh surface waves $-250 \mathrm{~m} / \mathrm{s}$ (this corresponds to a Poisson ratio of $\sigma=0.25$ ). The initial speed $v$ was $5 \mathrm{~m} / \mathrm{s}$, deceleration $-5 \mathrm{~m} / \mathrm{s}^{2}$, and the constant of ground attenuation of Rayleigh waves $\gamma=0.05$. The results for $V(\omega)$ (in $\mathrm{dB}$ relative to the reference level of $10^{-9} \mathrm{~m} / \mathrm{s}$ ) are shown on Figure 2 in the frequency range $0-30 \mathrm{~Hz}$.

One can see that curves corresponding to the approximate and exact values of $T_{x z}(\omega$, $k_{R} \cos \theta$ ) differ significantly from each other, especially at higher frequencies. The maxima of the "exact” curve are displaced towards higher frequencies. Apart from this, the exact approach does not generate deep zeros which occur in the approximate solution.

We must note, however, that most spectrum analysers used in environmental acoustics give readings averaged over certain frequency bands around the central frequency of 
interest - the so called octave, 1/2-octave and 1/3-octave spectra (see, e.g., Templeton et al. 1993).

Let us calculate 1/3-octave spectra, the narrowest of the above mentioned, for the approximate and exact solutions considered. Numerical calculation of these spectra (in $\mathrm{dB}$ relative to $10^{-9} \mathrm{~m} / \mathrm{s}$ ) may be carried out according to the formula

$$
V\left(\omega_{i}\right)=20 \log \left[\left(\sum_{j}\left|v_{z}\left(\omega_{i j}\right)\right|\right) /(2 P+1) \times 10^{-9}\right] .
$$

Here $\omega_{i}$ and $\omega_{i j}$ represent the vector and matrix of the discretised circular frequency, where index $i$ takes the values $i=1,2 \ldots N$, and index $j$ the values $j=1,2 \quad 2 P+1$. The vector of discretised frequency $\omega_{i}$ represents the values of the matrix $\omega_{i j}$ taken for $j=$ $P+1$. In the above expressions the number $N$ describes the upper boundary of the calculated frequency spectra: $F_{u p}=\omega_{u p} / 2 \pi=2^{\alpha N}$, where $\alpha$ is a constant of frequency discretisation: $F_{i}=\omega_{i} / 2 \pi=2^{\alpha i}$. The number $P$ is determined as $P=1 / 6 \alpha$, since for 1/3-octave spectra the relations between central and low-boundary frequencies, as well as between high boundary and central frequencies, are equal to $2^{1 / 6}$. In our calculations we used $\alpha=0.01$ that corresponded to $P=17$.

The comparison of the 1/3-octave spectra calculated for approximate and exact values of the Fourier integral for $T_{x z}\left(\omega, k_{R} \cos \theta\right)$ is shown on Figure 3. The values of all parameters are the same as on Figure 2. 
One can see that these spectra are almost identical. Thus, for calculations involving averaging over 1/3-octave frequency band one can use the simpler equation (15). Later on, we will give another convincing demonstration of this conclusion, when discussing the directivity patterns of generated ground vibrations.

\section{1/3-Octave Frequency Spectra}

Following the results of the previous section, we calculate the 1/3-octave spectra of vertical ground vibrations generated by braking vehicles using the simplified formula (equation (15)) and analyse their behaviour as functions of different parameters of the problem.

Figure 4 illustrates the behaviour of $1 / 3$ octave spectra of vertical ground vibrations generated by a braking lorry with $M=20000 \mathrm{~kg}$ for three different values of deceleration: $a=1,5$ and $9 \mathrm{~m} / \mathrm{s}^{2}$. The initial speed is $v=10 \mathrm{~m} / \mathrm{s}$. All other parameters are the same as on previous figures. It follows from Figure 4 that amplitudes of generated ground vibrations at all frequencies increase with increase of $a$. The behaviour of generated ground vibration spectra for three different values of the initial speed $(v=5$, 10 and $20 \mathrm{~m} / \mathrm{s}$ ) is shown on Figure 5 for $a=5 \mathrm{~m} / \mathrm{s}^{2}$ and $\Theta=\pi / 3$. It is seen that for frequencies higher than $4-5 \mathrm{~Hz}$ the spectra are almost independent of $v$. 
The above mentioned behaviour of the $1 / 3$-octave vertical ground vibration spectra is explained by the analysis of equation (15) which shows that for frequencies $F=\omega 2 \pi>>$ $a / v$ the ground vibration velocity is proportional to $a$ and does not depend on $v$, whereas for values of $F \leq a / v$ both these parameters, $a$ and $v$, cause oscillations of the ground vibration spectra.

The behaviour of generated ground vibrations (in $\mathrm{m} / \mathrm{s}$ ) as functions of acceleration $a$ and initial vehicle speed $v$ is illustrated respectively on Figures 6 and 7. The ground vibration velocities were averaged over 1/3-octave band around central frequencies $F=$ 4, 8, 16, 32 and $64 \mathrm{~Hz}$. Note that in agreement with the condition $F \leq a / v$ applied at fixed frequencies, oscillations of ground vibration level versus $a$ take place for larger $a$, whereas oscillations versus $v$ occur for smaller $v$. Also in agreement with the condition $F>>a / v$, the ground vibration velocity $v_{z}$ is proportional to a for higher frequencies and for lower accelerations (Figure 6) and does not depend on $v$ for higher frequencies and higher $v$ (Figure 7).

The linear dependence of ground vibration velocity on acceleration corresponds to its logarithmic dependence on dB-scale (see Figure 8). This implies that from the point of view of human perception, which is proportional to log-values of $V$, the most "sensitive" range of $a$ is from 0 to about $5 \mathrm{~m} / \mathrm{s}^{2}$ where ground vibrations increase very rapidly. After $a=5 \mathrm{~m} / \mathrm{s}^{2}$ the increase in ground vibrations with $a$ is slow. It is interesting that, according to Figure 7, for low-frequency components of ground vibration spectra (4 and 8 
$\mathrm{Hz}$ ) the level of generated vibrations may differ by about $12 \mathrm{~dB}$ if initial speed $v$ changes from 1 to $2 \mathrm{~m} / \mathrm{s}$. Thus, even small changes of $v$ in this range may result in large difference in ground vibrations generated in infrasound frequency range. This may cause high statistical deviations in levels of ground vibration generated by slowly approaching vehicles.

The influence of ground attenuation on 1/3-octave spectra of generated ground vibrations is shown on Figure 9 for three values of the attenuation parameter $(\gamma=0.05$, 0.1 and 0.15). All other parameters are the same as before. As expected, the higher values of $\gamma$ cause decreases of ground vibration level at higher frequencies.

For analysing the effect of Poisson ratio $\sigma$ on generated ground vibrations, the velocity of shear waves $c_{t}$ was determined as a function of $c_{l}$ and a Poisson ratio of $\sigma$ according to the well known relation $c_{t} / c_{l}=[(1-2 \sigma) / 2(1-\sigma)]^{1 / 2}$ (we recall that $\sigma$ can vary from 0 to 0.5 ). The velocity of Rayleigh surface waves $c_{R}$ was calculated as a function of $c_{t}$ and Poisson ratio according to the widely used approximate formula (see, e.g., Viktorov 1967) $c_{R} / c_{t}=(0.87+1.12 \sigma) /(1+\sigma)$. The results are shown on Figure 10 for $V$ averaged over 1/3-octave band around central frequencies $F=4,8,16,32$ and 64 Hz. Initial vehicle speed $v$ is $10 \mathrm{~m} / \mathrm{s}$, acceleration $a=5 \mathrm{~m} / \mathrm{s}^{2}$, ground attenuation $\gamma=$ 0.05. All other parameters are the same as on previous Figures.

According to Figure 10, for relatively low frequencies (4, 8 and $16 \mathrm{~Hz}$ ) the ground vibrations increase with increase of $\sigma$. However, for higher frequencies (32 and $64 \mathrm{~Hz}$ ) 
they strongly decrease with increase of $\sigma$. The latter is because, for higher frequencies of spectra, the influence of ground attenuation becomes overwhelming (see equation (12)). Since for $\sigma \rightarrow 0.5$ the Rayleigh wave velocity tends to zero, this results in a huge drop in ground vibration level.

According to the above calculations, typical levels of 1/3-octave frequency spectra of ground vibrations generated at the angle $\Theta=\pi / 3$ by heavy lorries $(M=20000 \mathrm{~kg})$ are around 40-50 dB (relative to $10^{-9} \mathrm{~m} / \mathrm{s}$ ). For the observation angle $\Theta=0$ the levels of vibration are around 46-56 dB. This is approximately of the same order as the averaged ground vibration velocity spectra generated by stationary traffic on uneven roads of very good quality (Hunt 1991).

\section{Directivity Patterns}

In contrast to the case of generating ground vibrations by vehicles travelling on uneven roads, for which the main mechanism is load forces directed normally to the ground surface, accelerating and braking vehicles are characterised by horizontal load forces that generate ground vibrations dependent on the observation angle $\Theta$ with respect to the vehicle movement. 
According to equation (12), and subject to the exact expression (14) for the load force $T_{x z}\left(\omega, k_{R} \cos \theta\right)$, the dependence of $V$ on $\Theta$ is determined both by a "dipole factor" $\cos \Theta$, following from the Green's function for horizontal load, and by exponential of the integral in equation (14). If one calculates angular dependence of ground vibration velocity using the traditional definition of directivity patterns in acoustics (e.g., Fahy 1993) as function $V(\Theta)$ taken at one particular frequency (in practical measurements this means using a very narrow-band spectral analysers), then it turns out that the influence of the exponential in equation (14) results in dramatic changes of directivity patterns even for small change in central frequency of a radiated spectrum.

Figure 11 shows the results of calculations of $V(\Theta)$ for two spectral components: $F=$ $12 \mathrm{~Hz}$ (a) and $F=13 \mathrm{~Hz}$ (b). Numerical calculations were carried out using equation (12) subject to the exact and approximate expressions for the load force $T_{x Z}\left(\omega, k_{R} \cos \theta\right)$, equations (14) and (15) respectively. The initial vehicle speed was $7 \mathrm{~m} / \mathrm{s}$, acceleration $5 \mathrm{~m} / \mathrm{s}^{2}$; other parameters are the same as on previous Figures.

Comparison of Figures 11a and 11b shows that directivities calculated according to equations (12) and (14) - dashed lines - are absolutely different from each other and both, being asymmetric versus the directions corresponding to $\Theta=\pi / 2$ and $3 \pi / 2$, differ from the symmetrical curve proportional to $\cos \Theta$ (solid lines) corresponding to the approximate approach of equations (12) and (15). 
Such a strong dependence of directivity pattern on frequency makes it impracticable to use a standard definition of directivity for the case considered. Instead, we calculate directivity patterns averaged in the 1/3-octave frequency range around the central frequencies 12 and $13 \mathrm{~Hz}$ using both exact and approximate approaches (equations (12), (14) and (12), (15) respectively).

The results are shown on Figure 12, a and b. One can see that calculations according to equations (12) and (14) averaged over 1/3-octave frequency band give at both frequencies the angular functions which are very close to $\cos \Theta$ corresponding to the approximate approach. This confirms once again the conclusion in Section 4 that for calculation of ground vibrations averaged over 1/3-octave frequency band, the approximate approach neglecting the terms with $v$ and $a$ in equation (14) gives almost the same results as the more time consuming direct approach which involves numerical integration of equation (14).

Thus, the directivity patterns of ground vibrations from accelerated or braking vehicles averaged over 1/3-octave frequency band do not depend on the direction of vehicle movement and are described by a dipole factor $\cos \Theta$. It is evident that there is no radiation at the angles $\Theta=\pi / 2$ and $3 \pi / 2$, i.e., in the directions perpendicular to the vehicle movement. The presence of a dipole factor reflects the reduction of symmetry in the problem under consideration due to the action of horizontal forces. 


\section{Conclusions}

Generation of ground vibrations by accelerating and braking vehicles has been considered using the Green's function method in the frequency domain. The resulting ground vibration field has been calculated for the far field of radiation taking into account only the Rayleigh surface wave contribution.

Two approaches to the calculation were considered: the approximate approach that ignores the changing location of the vehicle during acceleration (deceleration), and a more exact one that takes them into account. It was shown that, although ground vibration velocities $V$ calculated using these approaches are significantly different if rigorous (narrow band) Fourier spectra are being calculated, they become almost identical in terms of 1/3-octave averaged spectra traditionally used in practical measurements in environmental acoustics. This allows practical calculations using the simpler approximate expressions.

The 1/3-octave frequency spectra of ground vibrations generated by heavy lorries $(M=20000 \mathrm{~kg})$, have been calculated and investigated as functions of the acceleration $\pm a$, final or initial speed of the vehicle $v$, ground attenuation coefficient $\gamma$, Poisson ratio $\sigma$, and radiation angle relative to the direction of the vehicle movement $\Theta$. It was shown that the amplitudes of ground vibration spectra generated by accelerating and braking 
vehicles are identical. It is also shown that for medium and upper bands of the spectra the generated ground vibration level is determined mainly by the value of acceleration $|a|$ and is almost independent of the initial (final) vehicle speed $v$; whereas for low band oscillations of ground vibration amplitude versus both $a$ and $v$ may take place that may result in large statistical deviations of experimentally observed data. It is shown that typical levels of 1/3-octave frequency spectra of ground vibrations generated at the angle $\Theta=\pi / 3$ by heavy lorries $\left(M=20000 \mathrm{~kg}\right.$ ) are around $40-50 \mathrm{~dB}$ (relative to $10^{-9} \mathrm{~m} / \mathrm{s}$ ). At the angle of maximum radiation $\Theta=0$ the levels of generated vibrations are around 46$56 \mathrm{~dB}$. This is approximately of the same order as the averaged ground vibration velocity spectra generated by stationary traffic on uneven roads of very good quality.

In contrast to vehicles travelling along uneven roads that generate vibrations propagating at all directions along the ground surface, the ground vibrations from accelerating and braking vehicles are characterised by the directivity function. For ground vibration amplitudes averaged over the 1/3-octave frequency band this function is simply $\cos \Theta$ which shows that there is no radiation in the directions perpendicular to the direction of vehicle movement.

The results obtained in this paper may be used in practice for the prediction of ground vibrations generated by accelerating and braking road vehicles around the sites where frequent stopping and starting is most likely, e.g., near traffic lights, petrol stations, or sites of loading and unloading. 


\section{References}

Beitin, K.I., 1969, "Response of anelastic half space to a decelerating surface point load”, Transactions of the ASME: Journal of Applied Mechanics, December 819-826.

Ewing, W.M, Jardetzky, W.S. and Press, F., 1957, Elastic waves in layered media, McGraw-Hill, New York.

Fahy, F., 1993, Sound and structural vibration, Academic Press, London.

Graff, K.F., 1975, Wave motion in elastic solids, Clarendon Press, Oxford, United Kingdom.

Gutovski, T.G. and Dym, C.L., 1976, "Propagation of ground vibration: a review”, Journal of Sound and Vibration 49, 179-193.

Hanazato, T., Ugai, K., Mori, M., and Sakaguchi, R., 1991, “Three-dimensional analysis of traffic-induced ground vibrations”, Journal of Geotechnical Engineering 117, 11331151. 
Hunt, H.E.M., 1991, "Stochastic modelling of traffic-induced ground vibrations”, Journal of Sound and Vibration 144, 53-70.

Krylov, V.V., 1995, “Generation of ground vibrations by superfast trains”, Applied Acoustics 44, 149-164.

Krylov, V., and Ferguson, C., 1994, "Calculation of low-frequency ground vibrations from railway trains”, Applied Acoustics 42, 199-213.

Lamb, H., 1904, “On the propagation of tremors over the surface of an elastic solid”, Philosophical Transactions of the Royal Society, London A203, 1-42.

Le Houedec, D. and Riou, Y., 1982, “Analytic response of a semi-infinite soil mass to road traffic vibration”, in Proceedings of the Conference on Soil Dynamics and Eartquake Engineering, Southampton, 209-233.

Newcomb, T.P., and Spurr, R.T., 1967, Braking of road vehicles, Chapman and Hall, London. 
Taniguchi, E. and Sawada, K., 1979, “Attenuation with distance of traffic-induced vibrations”, Japanese Society of Soil Mechanics and Foundation Engineering: Soils and Foundations 19, 15-28..

Templeton, D., Sacre, P., Mapp, P. and Saunders, D., 1993, Acoustics in the built environment, Butterworth Architecture, Oxford, United Kingdom.

Viktorov, I.A., 1967, Rayleigh and Lamb waves, Plenum Press, New York.

Watts, G.R., 1984, "Vibration nuisance from road traffic - results of a 50 site survey”, Transport Research Laboratory report LR1119 (Crowthorne, United Kingdom).

Wong, J.Y., 1993, Theory of ground vehicles, John Wiley \& Sons, New York. 


\section{Figure captions}

Figure 1. Schematic diagram of the problem

Figure 2. Comparison of "narrow-band” vertical ground vibration spectra calculated using approximate and exact computations of the Fourier integral for a vehicle load force - curves $V 1$ and $V 2$ respectively

Figure 3. Comparison of 1/3-octave vertical ground vibration spectra calculated using approximate and exact computations of the Fourier integral for a vehicle load force - curves $V 1$ and $V 2$ respectively

Figure 4. 1/3-octave vertical ground vibration spectra calculated for three values of vehicle acceleration (deceleration) $a: a=1 \mathrm{~m} / \mathrm{s}^{2} \quad$ (curve $V 1$ ), $a=5 \mathrm{~m} / \mathrm{s}^{2}$ (curve $V 2$ ) and $a=9 \mathrm{~m} / \mathrm{s}^{2}$ (curve $V 3$ ) 
Figure 5. 1/3-octave vertical ground vibration spectra calculated for three values of final (initial) vehicle speed $v: \quad \mathrm{v}=5 \mathrm{~m} / \mathrm{s} \quad$ (curve $V 1$ ), $v=10 \mathrm{~m} / \mathrm{s}$ (curve $V 2$ ) and $v$ $=20 \mathrm{~m} / \mathrm{s}$ (curve $\mathrm{V3}$ )

Figure 6. Vertical ground vibration amplitudes (in $\mathrm{m} / \mathrm{s}$ ) averaged over 1/3-octave frequency band as functions of vehicle acceleration (deceleration) $a$ for the central frequencies $F=4,8,16,32$ and $64 \mathrm{~Hz}$ - curves $V 1, V 2, V 3, V 4$ and V5 respectively

Figure 7. Vertical ground vibration amplitudes (in $\mathrm{m} / \mathrm{s}$ ) averaged over 1/3-octave frequency band as functions of final (initial) vehicle speed $v$ for the central frequencies $F=4,8,16,32$ and $64 \mathrm{~Hz}$ - curves $V 1, V 2, V 3, V 4$ and $V 5$ respectively

Figure 8. The same as on Fig.6 but using dB-scale for ground vibration amplitudes

Figure 9. Effect of ground attenuation $\gamma$ on 1/3-octave vertical ground vibration spectra: $\gamma$ $=0.05$ (curve V1), $\gamma=0.10$ (curve V2) and $\gamma=0.15$ (curve V3) 
Figure 10. Vertical ground vibration amplitudes averaged over 1/3-octave frequency band as functions of the Poisson ratio $\sigma$ for the central frequencies $F=4,8,16,32$ and $64 \mathrm{~Hz}$ - curves $V 1, V 2, V 3, V 4$ and $V 5$ respectively

Figure 11. Comparison of "narrow-band" radiation patterns of ground vibrations for the spectral components $F=12 \mathrm{~Hz}$ (a) and $F=13 \mathrm{~Hz}$ (b) calculated using approximate and exact approaches to computation of the Fourier integral for the vehicle load force - curves V1 and V2 respectively

Figure 12. Comparison of “1/3-octave averaged” radiation patterns of ground vibrations for the spectral components $F=12 \mathrm{~Hz}$ (a) and $F=13 \mathrm{~Hz}$ (b) calculated using approximate and exact approaches to computation of the Fourier integral for the vehicle load force - curves $V 1$ and $V 2$ respectively 


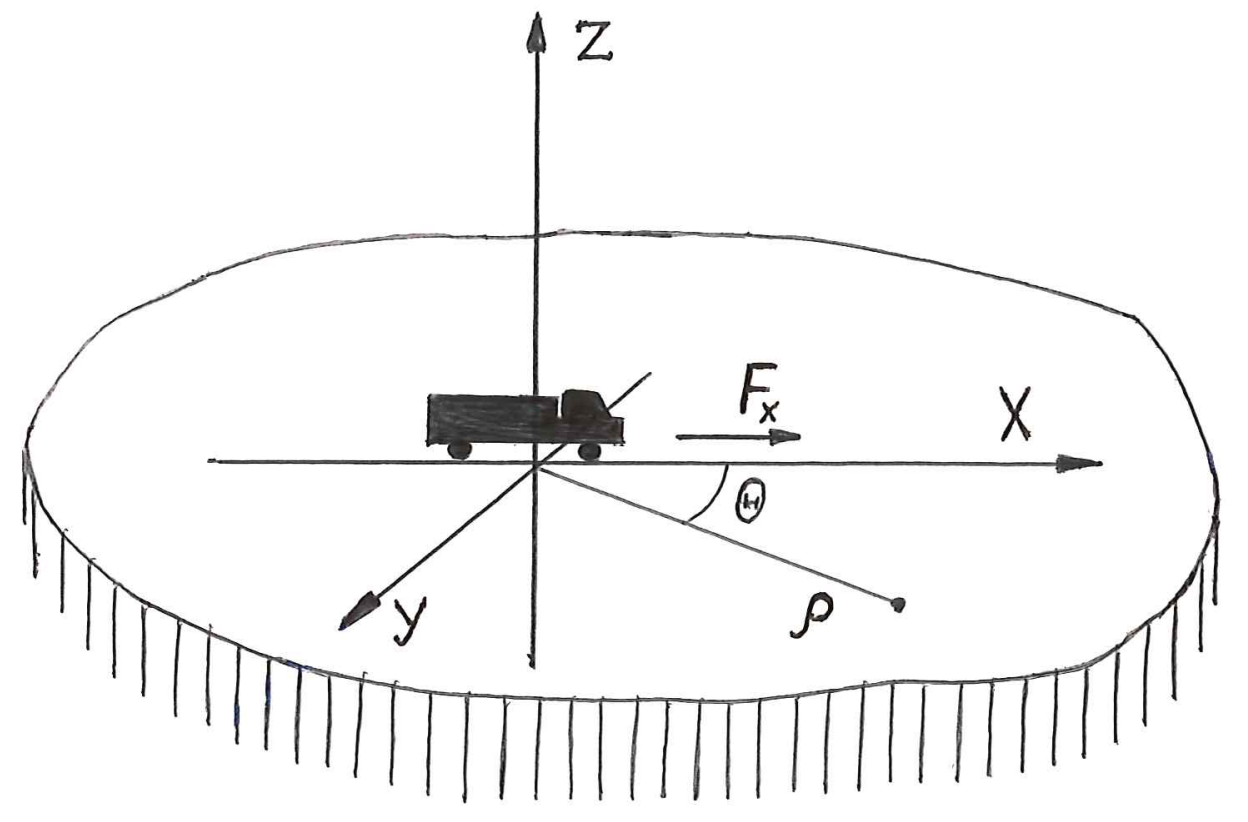

Fig. 1 


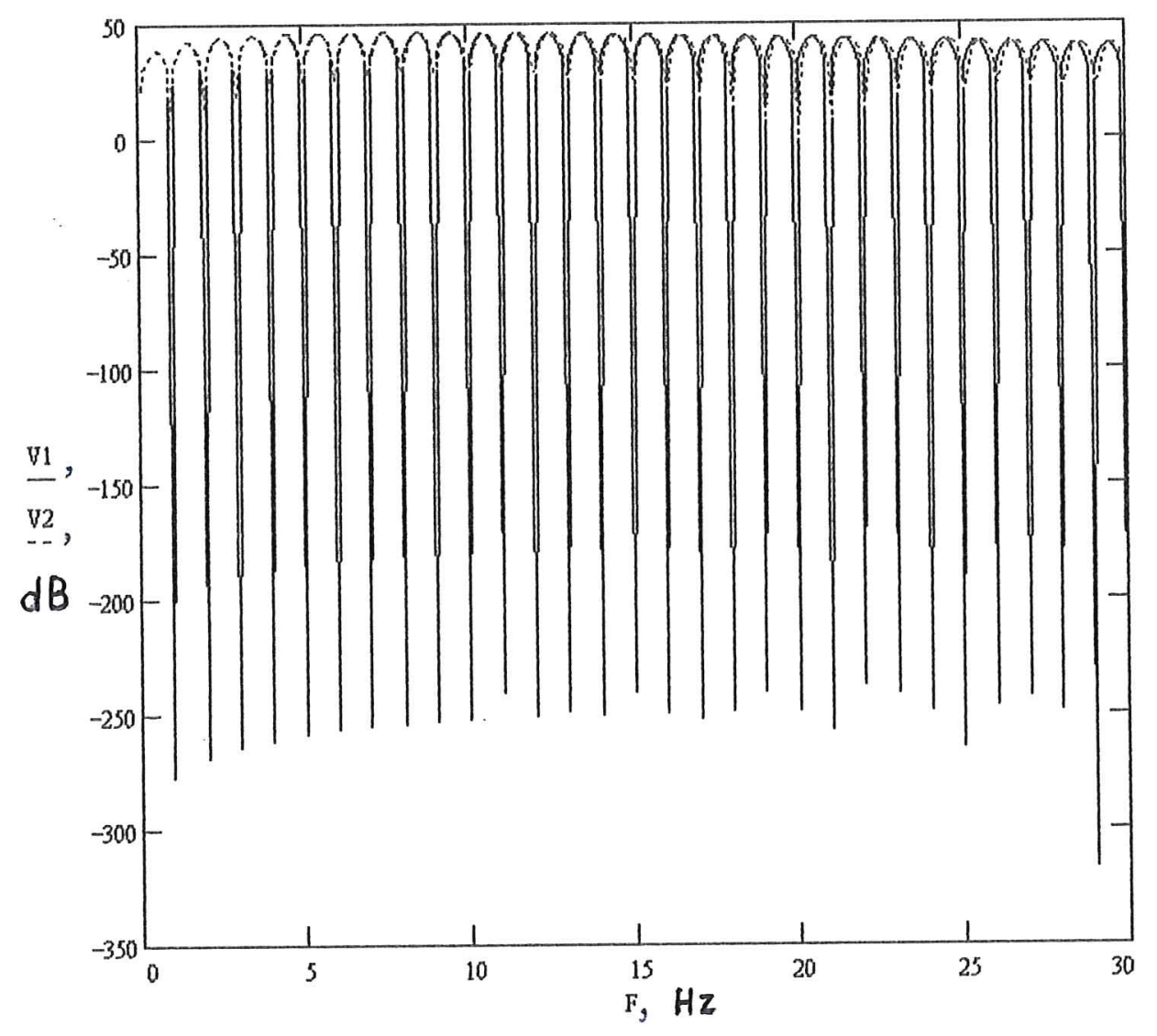

Fig. 2 


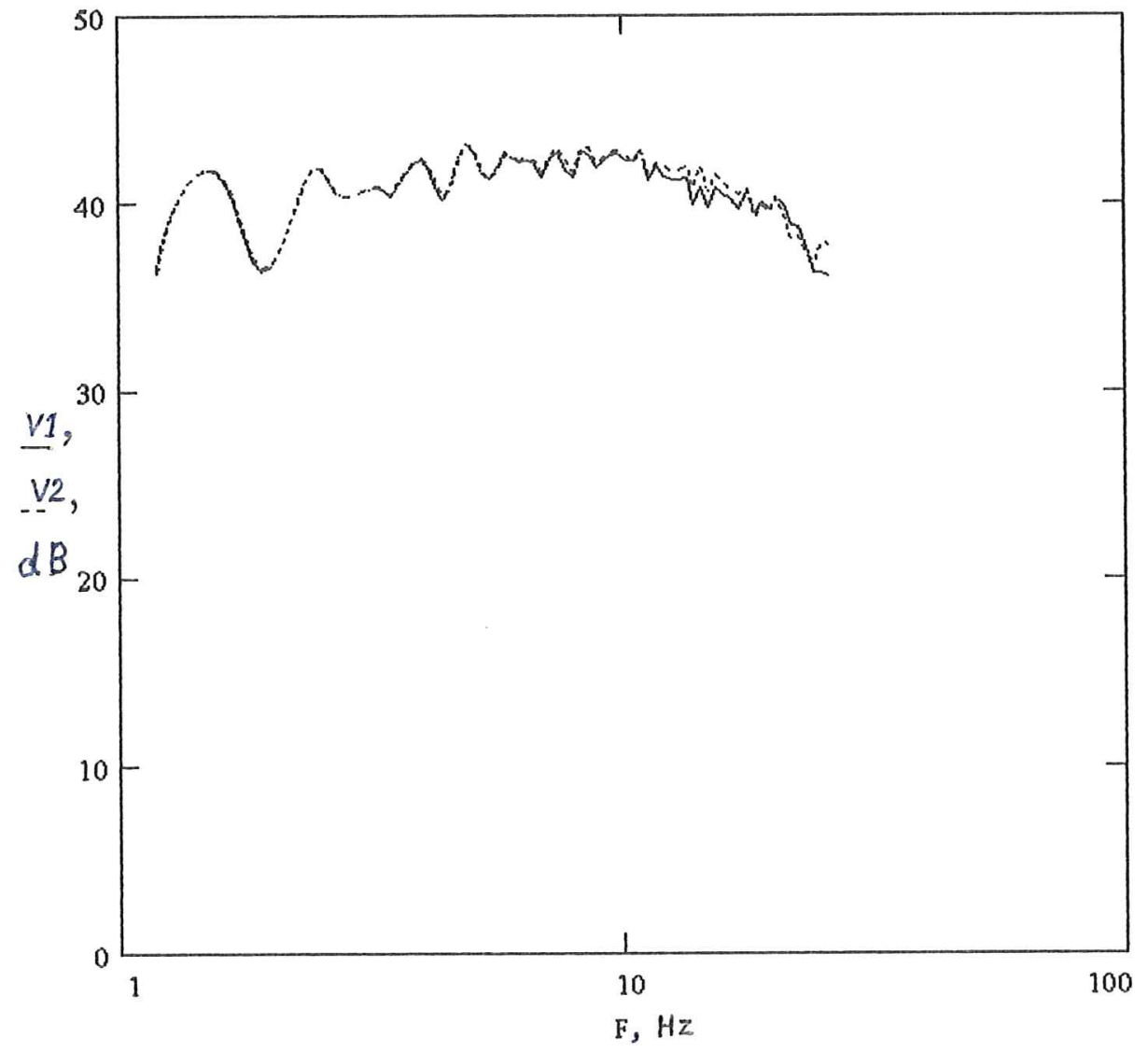

Fig. 3 


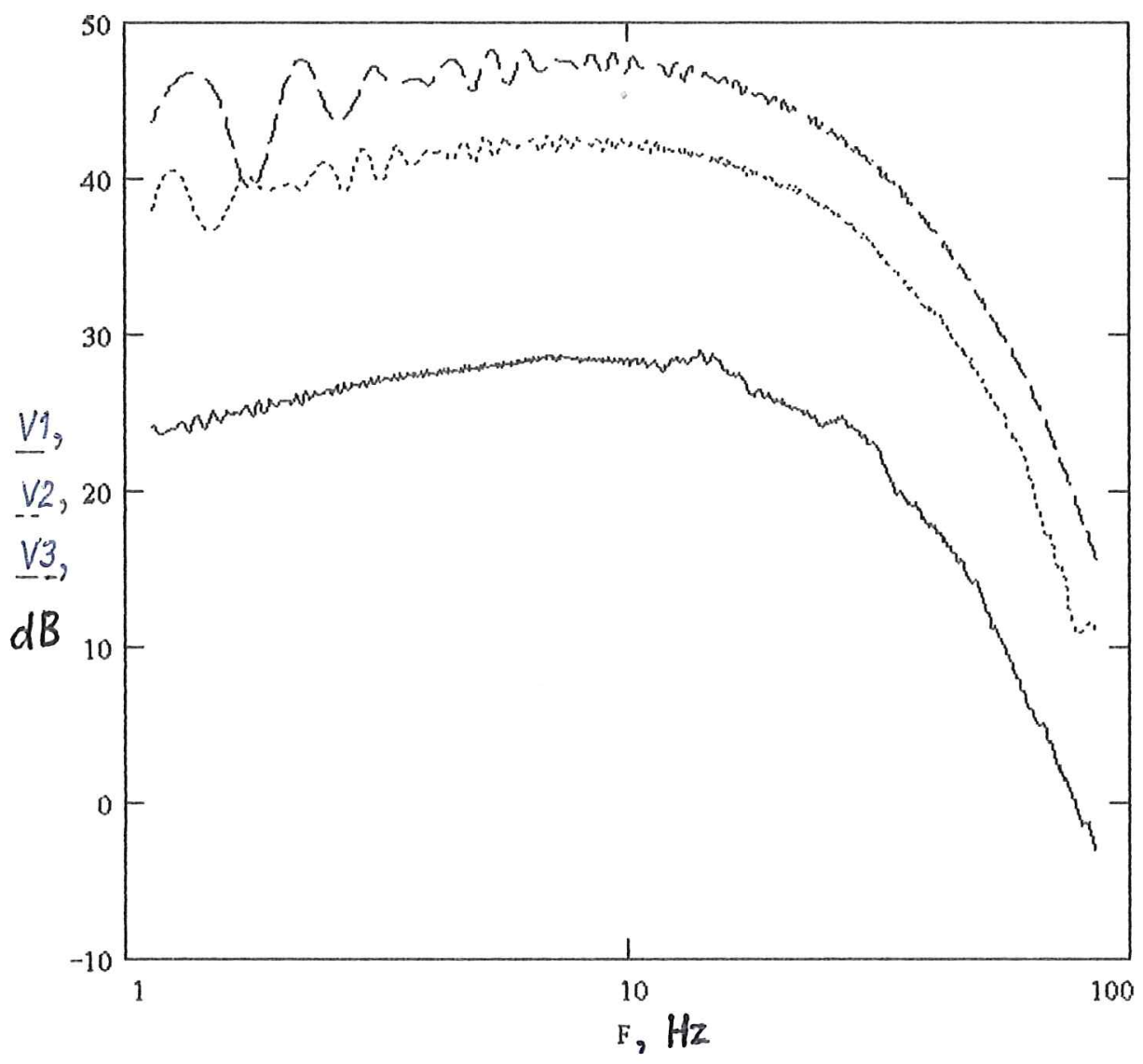

Fig. 4 


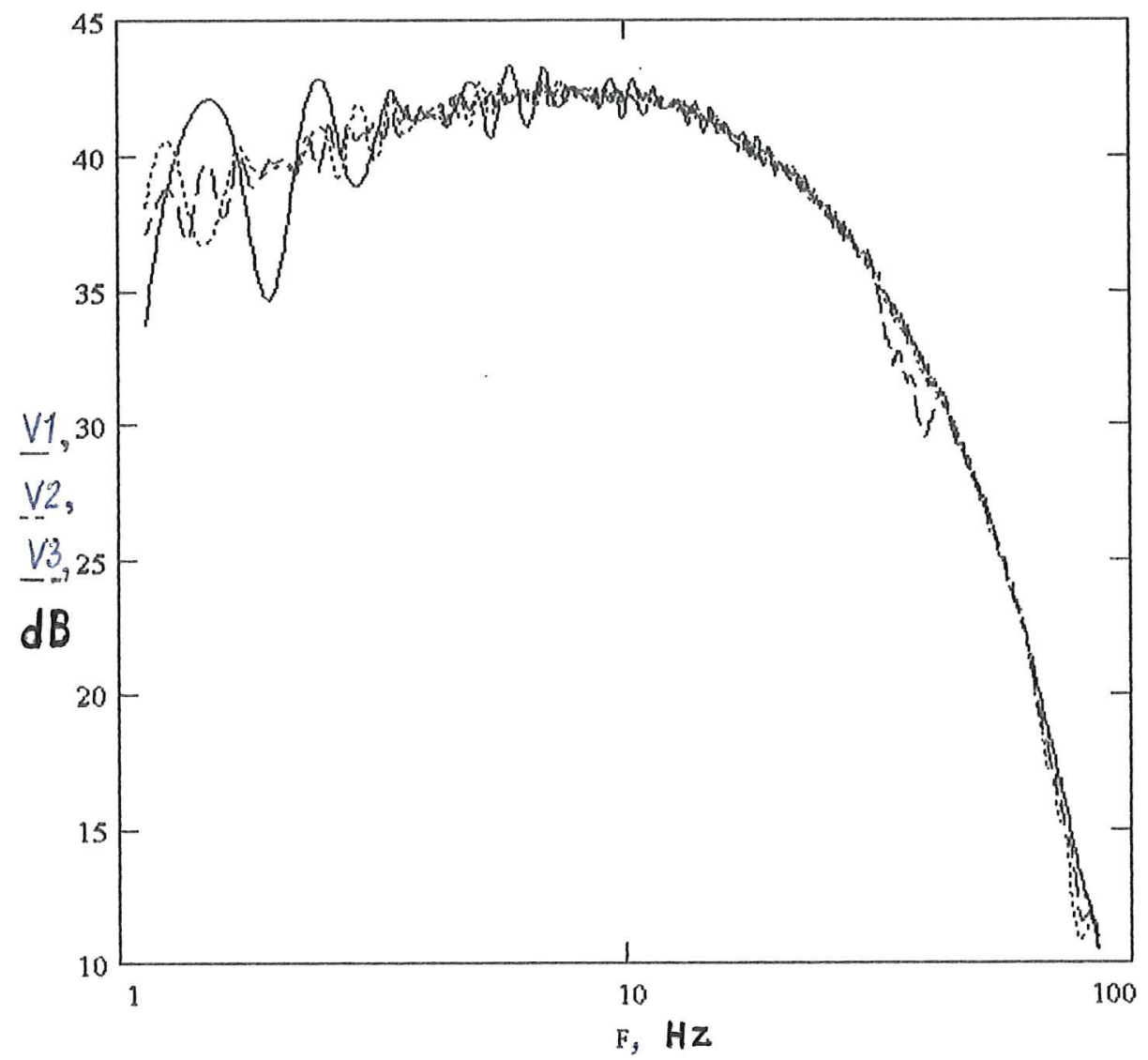

$$
\text { Fig. } 5
$$




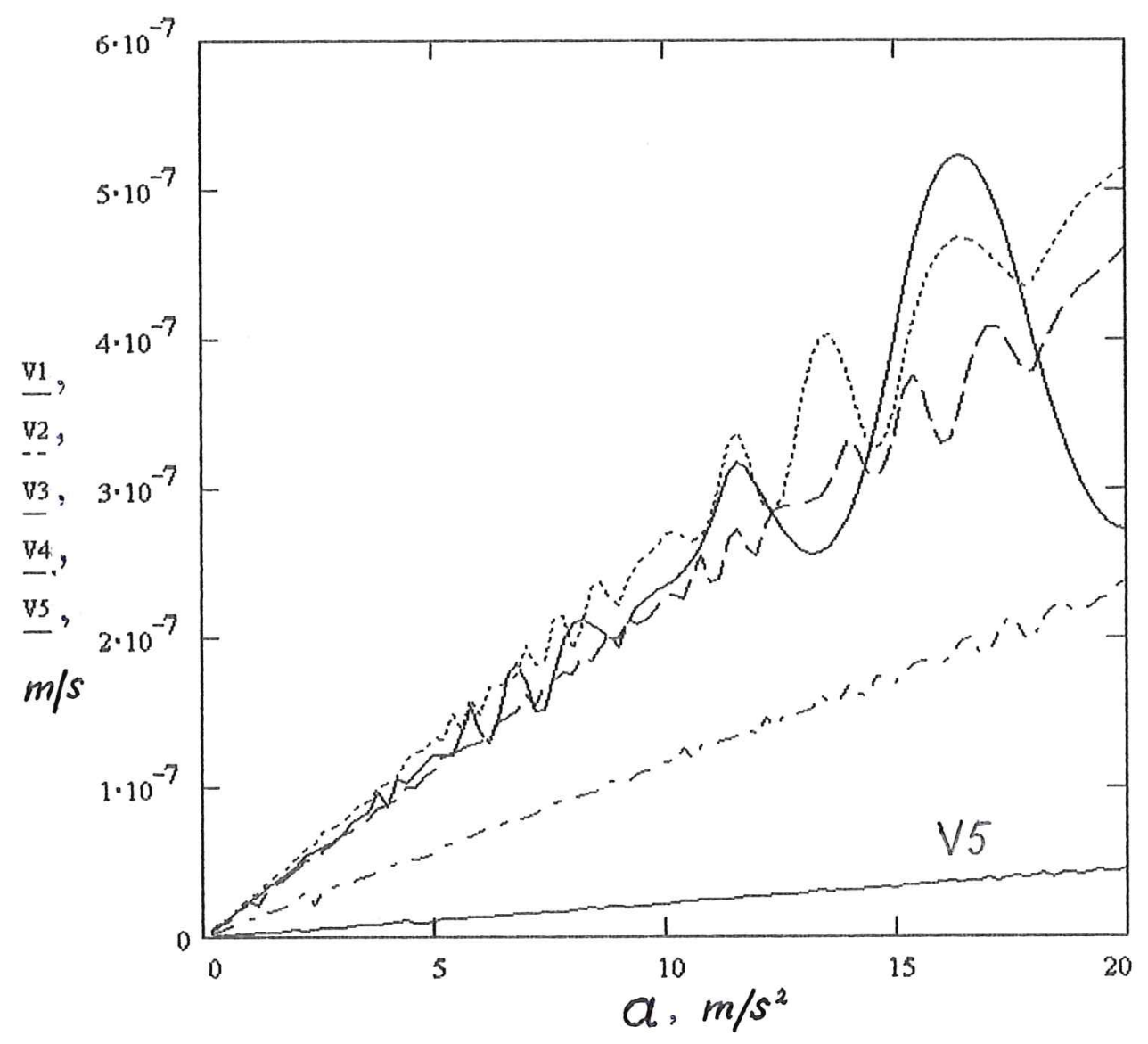

Fig. 6 


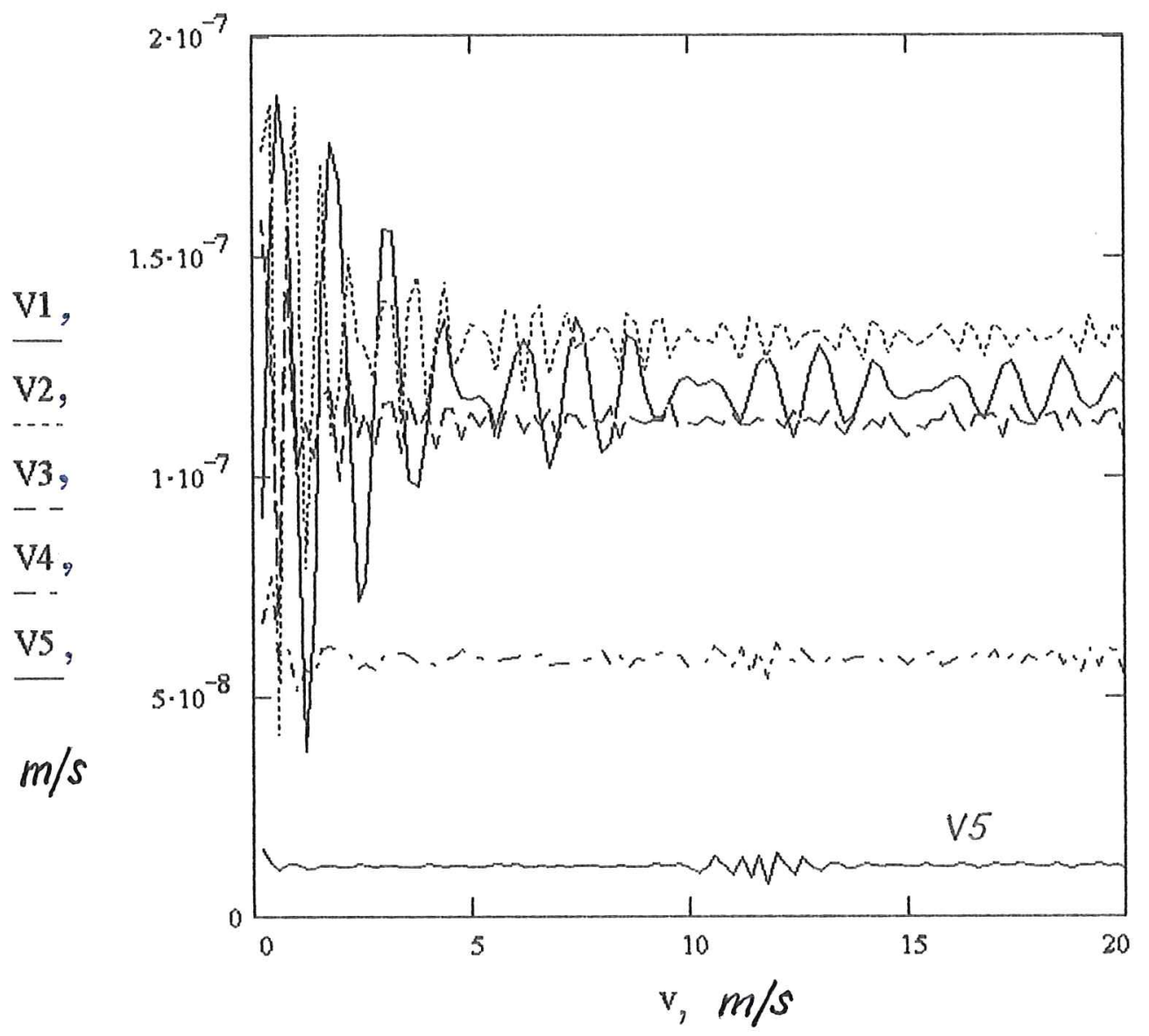

$$
\text { Fig. } 7
$$




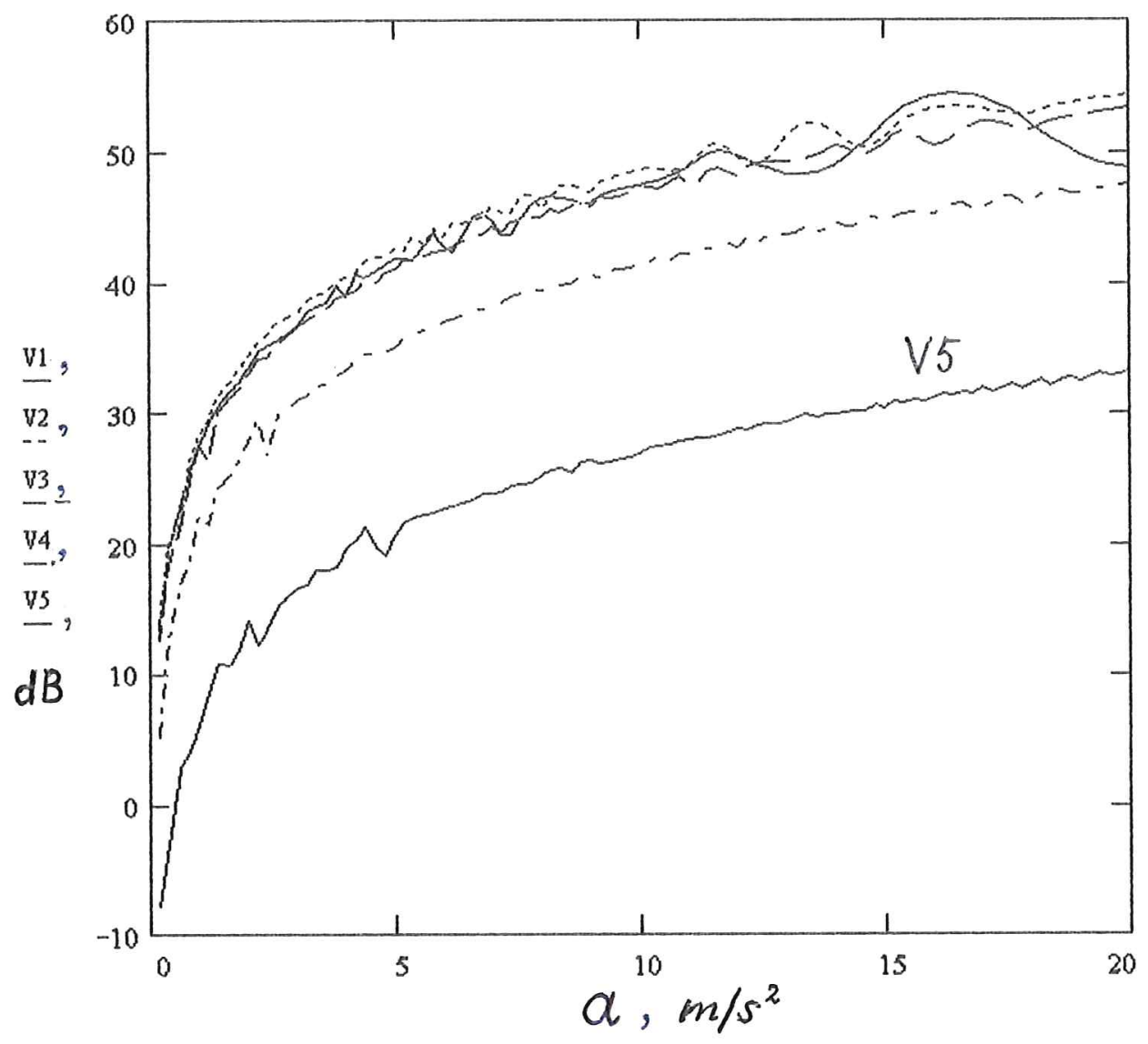

Fig. 8 


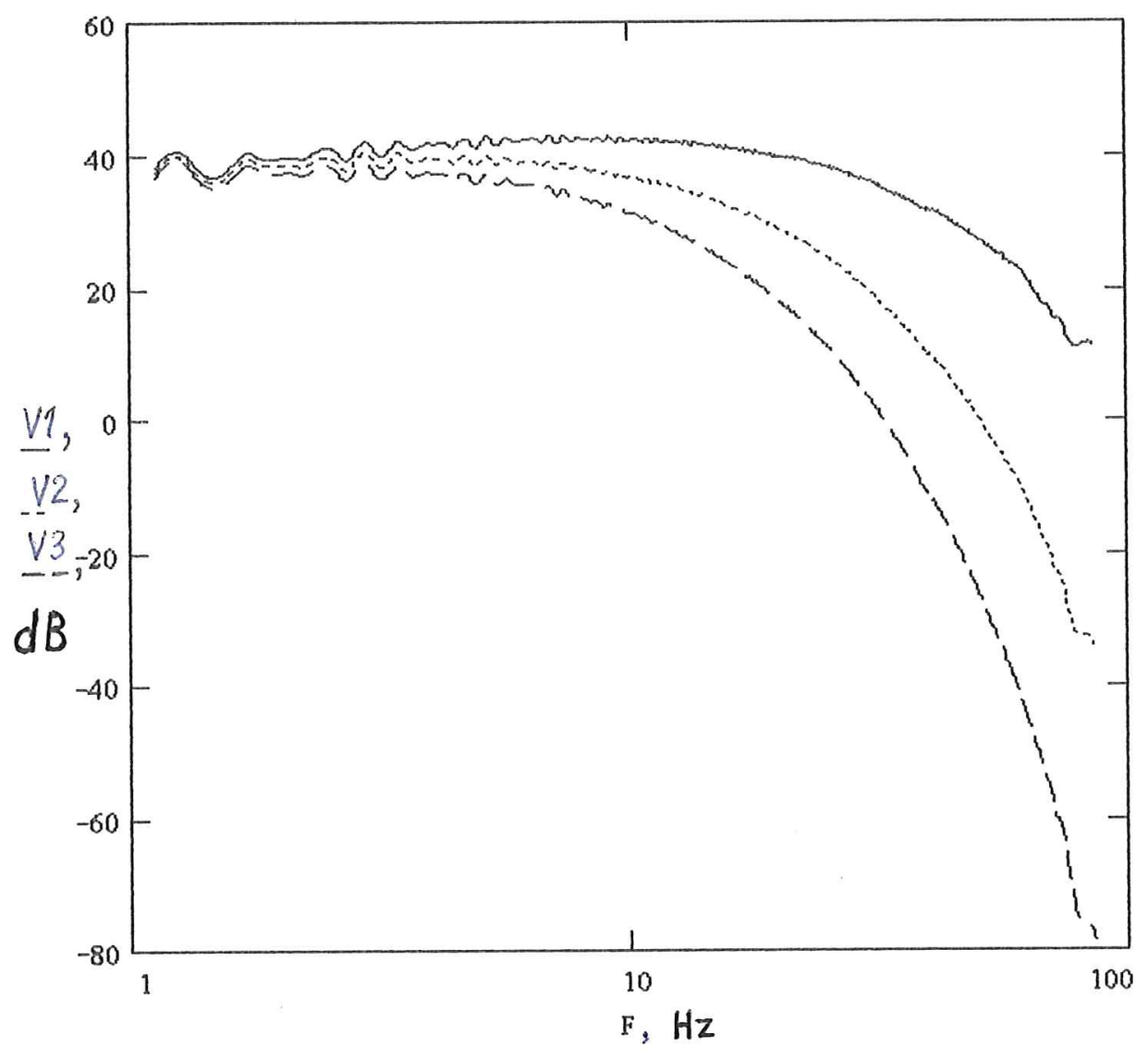

Fig. $g$ 


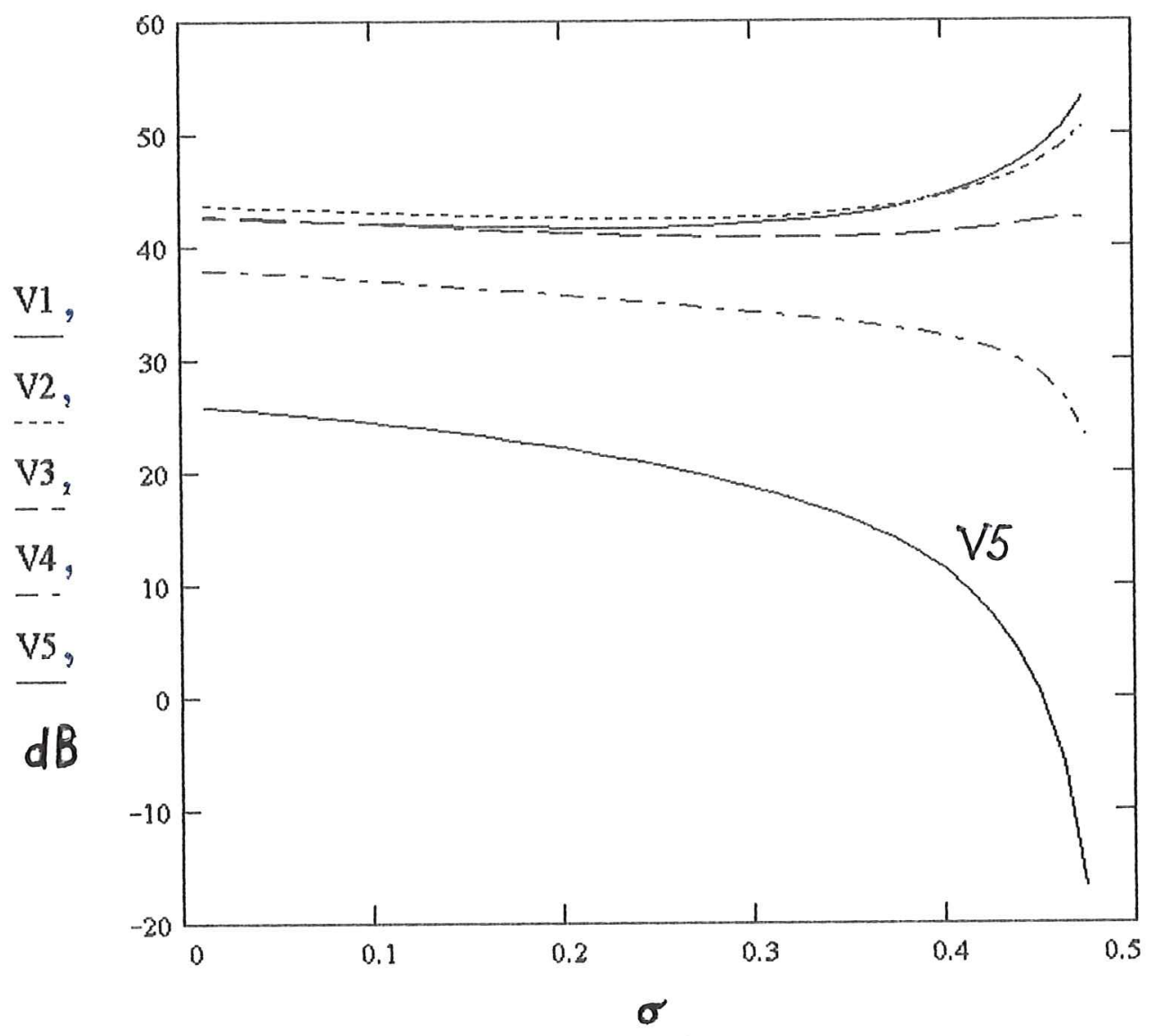

Fig. 10 


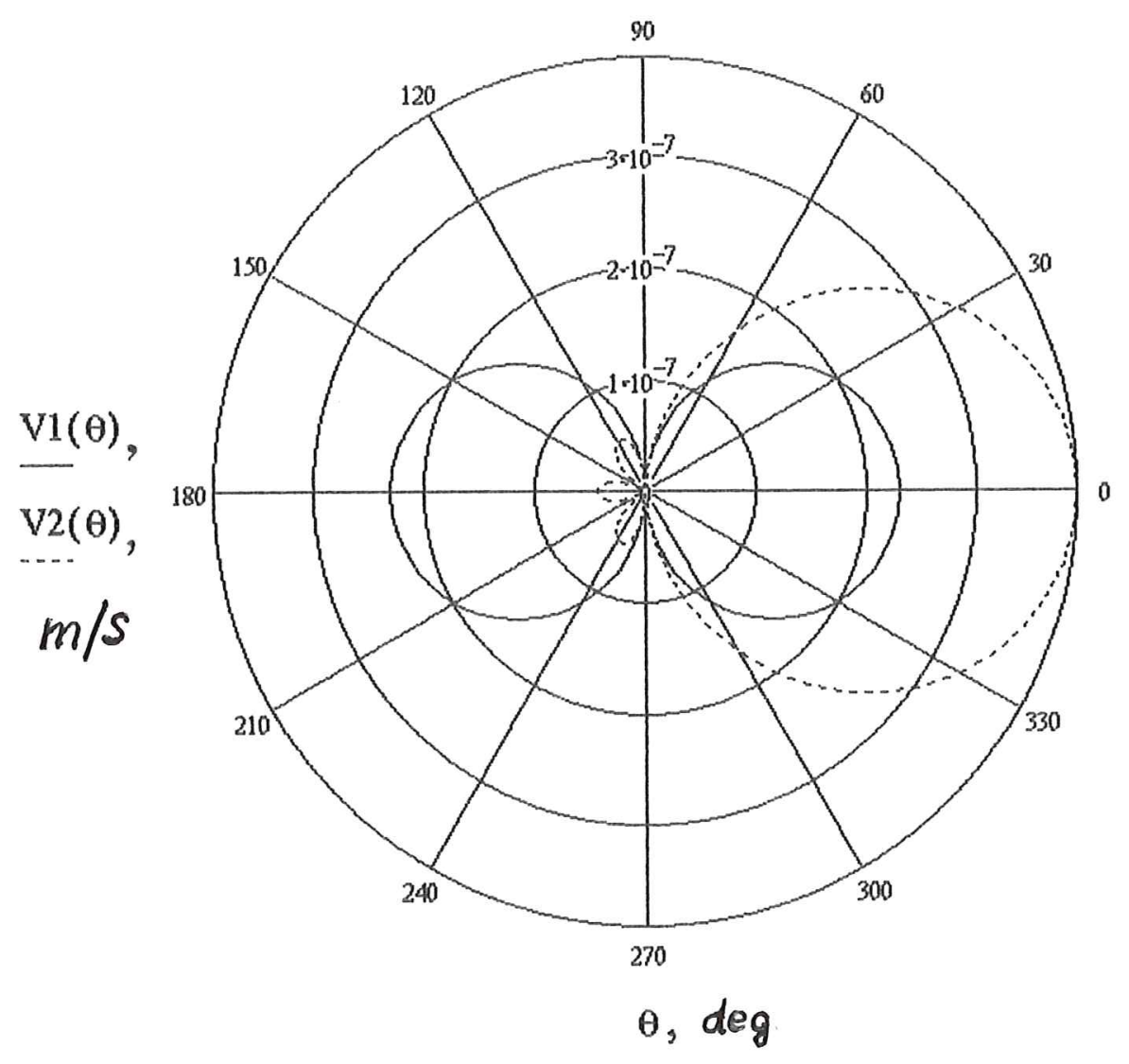

Fig. $11 \alpha$ 


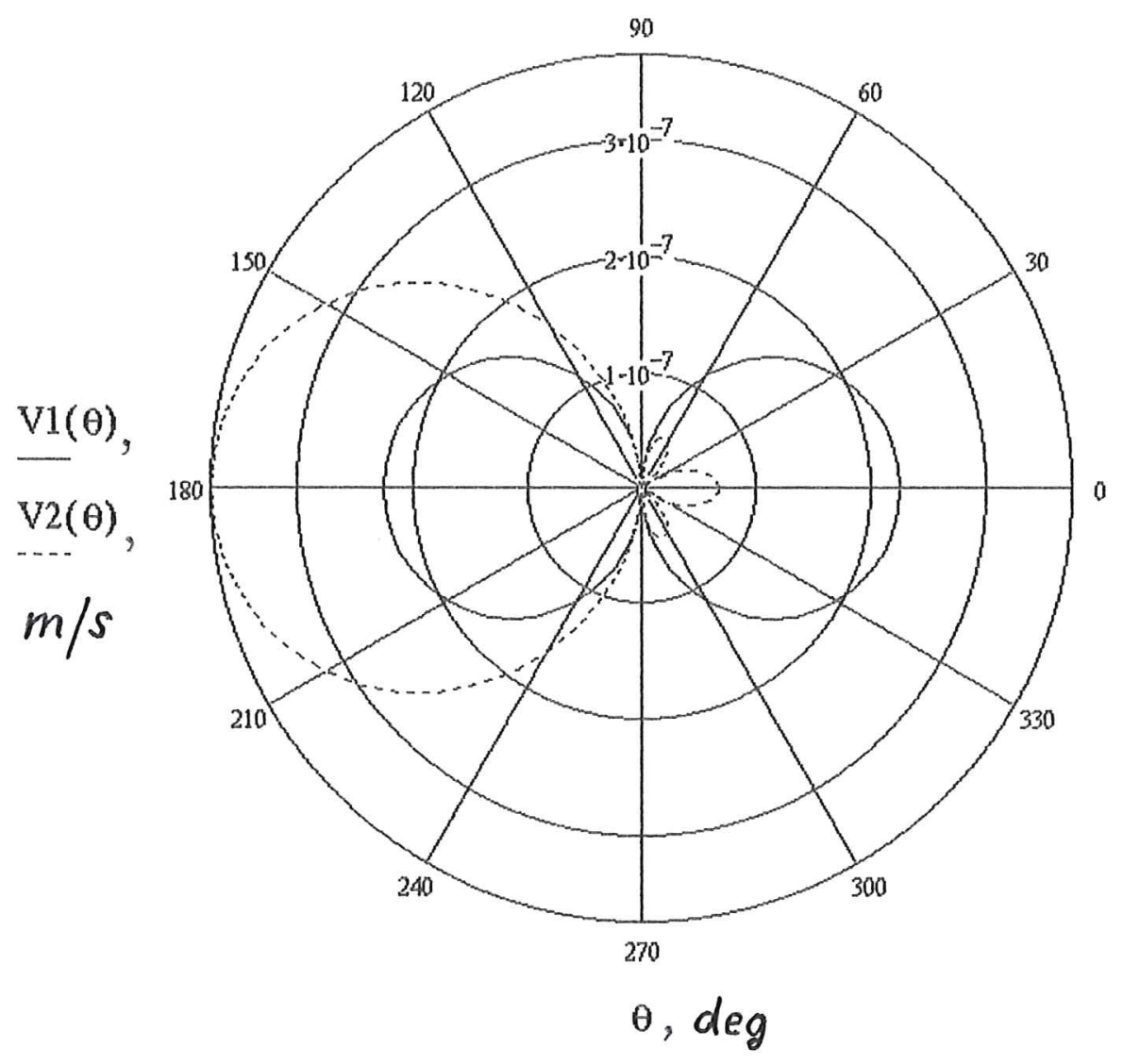

Fig. 116 
$a$

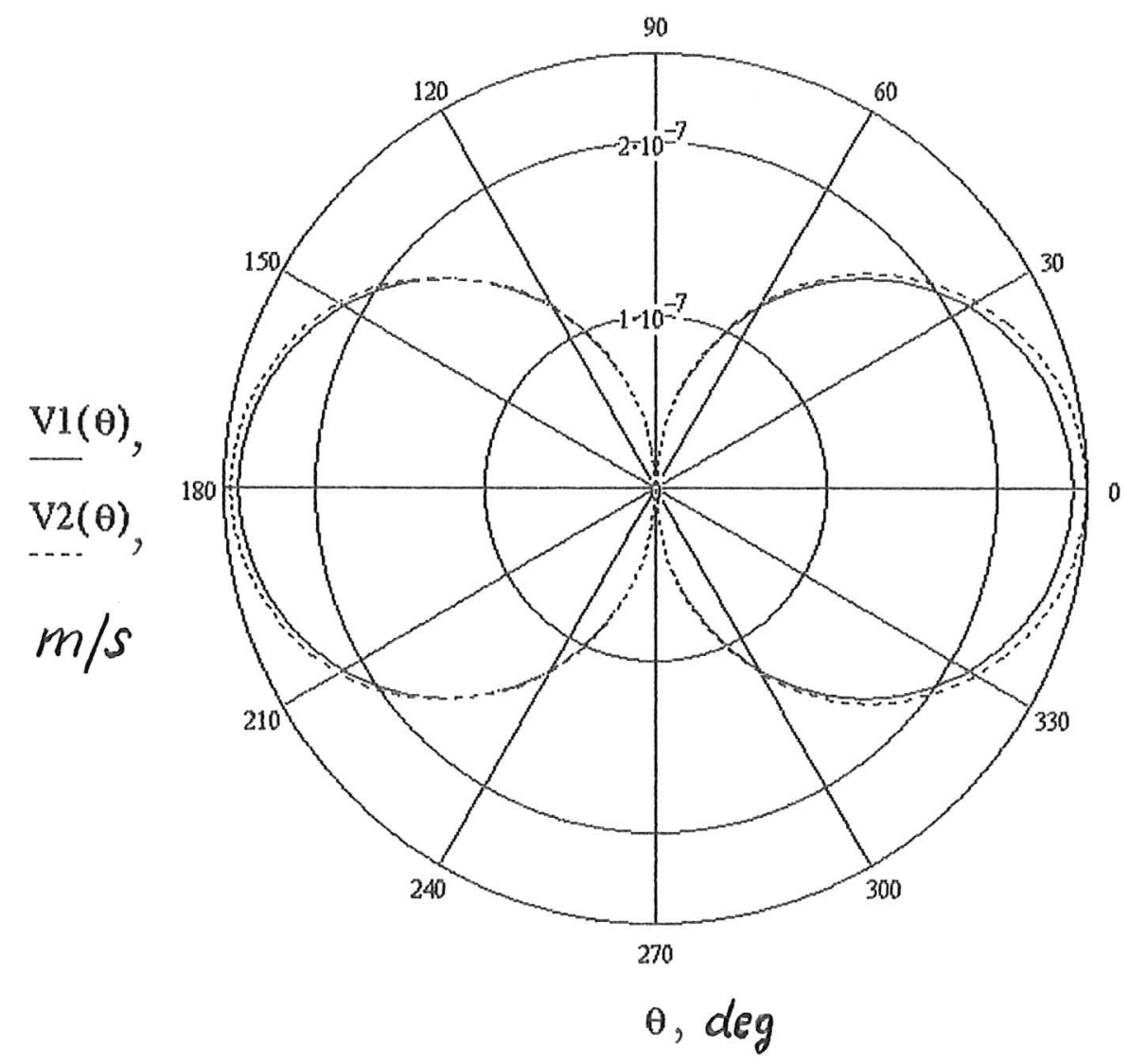

Fig. $12 a$ 


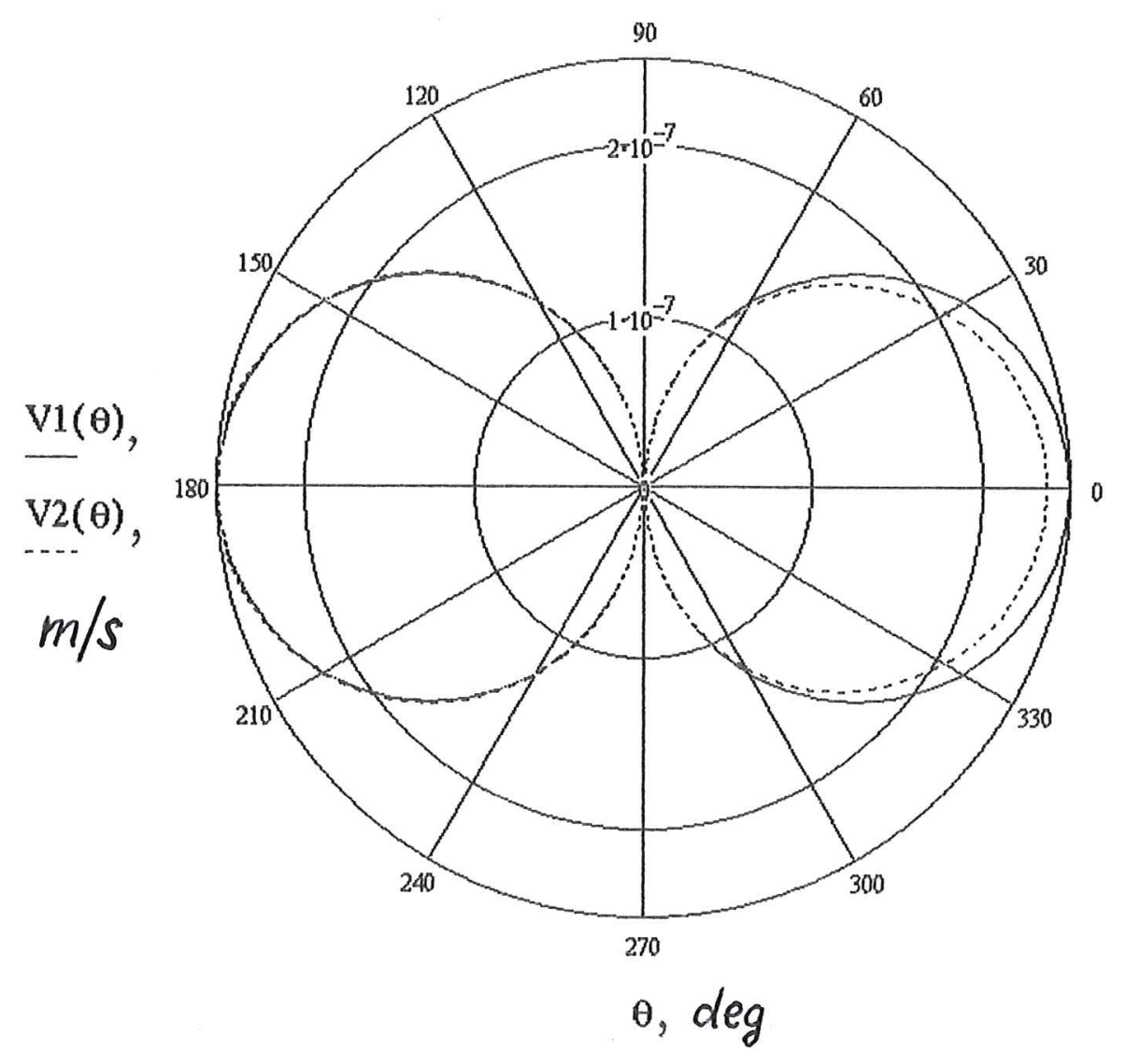

Fig. $12 b$ 